\title{
RASTREIO DO CÂNCER DE PRÓSTATA: REVISÃO SISTEMÁTICA DA LITERATURA SOBRE AS PERSPECTIVAS MUNDIAIS
}

\author{
PROSTATE CANCER SCREENING: SYSTEMATIC LITERATURE REVIEW OF WORLDWIDE PERSPEC- \\ TIVES
}

\section{Julio Cesar Betiola , Cristiano Dias do Nascimentob, Flávio Aparecido dos Reis da Silvac, Valter Carabetta Júniord, Maurício Hachule}

ajbetiol@usp.br, bcristiano.fmsa@gmail.com, cflavio.fmsa@gmail.com, dvcarabetta@prof.unisa.br, emhachul@prof.unisa.br Universidade de Santo Amaro - São Paulo (SP), Brasil

\section{RESUMO}

Introdução: $\mathrm{Na}$ última década surgiu um expressivo volume de debates relacionados ao rastreio do câncer de próstata, sendo os principais tópicos discutidos: quando; em quem e como utilizar os níveis de PSA e/ou Toque Retal em um contexto populacional. Uma vez que diferentes instituiçóes, órgãos governamentais e RCT fornecem recomendaçóes distintas e, em certos casos, controversas, tentativas de organizá-las são necessárias. Objetivo: $\mathrm{O}$ estudo visou avaliar o atual contexto das recomendaçóes relacionadas ao rastreio massivo, seletivo ou oportunista do câncer de próstata, utilizando os níveis de PSA e/ou Toque Retal. Materiais e métodos: Revisão narrativa de literatura em bases de dados: MEDLINE, LILACS, Embase e Cochrane Library. Utilizaram-se termos de pesquisa pré-estabelecidos na Universidade de Santo Amaro (Unisa), São Paulo, Brasil. A seleção inicial baseou-se em títulos e resumos dos artigos. A análise integral e avaliação crítica de amplos RCT prospectivos, meta-análises abrangentes, forças-tarefas nacionais e recomendaçôes de organizaçóes médicas foram realizadas. Resultados: A estratégia de pesquisa gerou 1.046 citaçóes. Treze recomendaçóes tiveram seus níveis de evidência e pontos de divergência ou concordância avaliados. Conclusão: Os RCT existentes apresentam níveis insuficientes de concordância, os quais embasam diretrizes governamentais. Algumas recomendaçōes mundiais são sumarizadas em opostos extremos. Até que estudos com alto grau de recomendação surjam, determinando metodologia e grupos que se beneficiariam do rastreio, a decisão compartilhada em pacientes bem informados aparenta ser atualmente uma recomendaçáo apropriada para o rastreio populacional do câncer de próstata, tanto que, por hora, o rastreio em massa de população deve ser abandonado como política pública de saúde.

Palavras-chave: Próstata; neoplasia; programas de rastreamento; PSA; exame retal digital.

\section{ABSTRACT}

Introduction: Over the last decade increased the debate regarding prostate cancer screening and one of the main discussed topics were: when, in whom, and how to use PSA levels and/or DRE in a population based level. Once different institutions, government and RCT's provide distinct, and sometimes, controversial recommendations, an attempt to summarize these studies is valuable. Aim: The study aimed to assess the actual state of the art of recommendations regarding the use of routine mass, selective, or opportunistic screening for prostate cancer with PSA levels and/or DRE. Material and methods: A narrative review of the literature was undertaken through MEDLINE, LILACS, Embase and Cochrane Library database, using pre-established search terms at University of Santo Amaro (Unisa), São Paulo, Brazil. Initial selection was performed based on title and abstracts assessment. Full-text analysis and critical appraisal of large, prospective RCT, comprehensive meta-analysis, National Taskforces, Medical and National organizations recommendations were accomplished. Results: Search strategy yielded 1046 citations. 
Thirteen recommendations had their levels of evidence and points of divergence or agreement evaluated. Conclusion: Existing RCT's exhibit insufficient levels of agreement and additionally governmental guidelines are based on them. Some worldwide recommendations are currently summarized in opposite extremes. Until high grade of recommendation studies determine the best methodology and group of population that would benefit from screening, the shared decision-making in well-informed individuals currently seems to be an appropriated recommendation regarding the population screening of prostate cancer and, by now, it seems reasonable to forgo mass screening as a public heatlh policy.

Keywords: Prostate; neoplasia; screening; PSA; digital rectal examination.

\section{Introdução}

As políticas públicas brasileiras historicamente privilegiaram programas orientados à saúde de mulheres e crianças. O movimento feminista na década de 1970 demandou açóes governamentais, as quais instituíram em 1984 o Programa de Assistência Integral à Saúde da Mulher (PAISM) ${ }^{1}$. Somente em 2008 o governo lançou uma política pública específica aos homens, o PNAISH (Política Nacional de Atenção Integral à Saúde do Homem), visando reduzir a morbimortalidade masculina via expansão e facilitação do acesso, prevenção e açóes de cuidado a esse grupo ${ }^{2}$. De acordo com Carrara et al. ${ }^{3}$, o PNAISH foi elencado pelo ministro da Saúde como uma política pública pioneira a nível mundial, sendo a primeira na América Latina e a segunda nas Américas, posterior apenas ao Canadá. Segundo os dados da Projeção da População (2000-2060) ${ }^{4}$, em 2016, a populaçáo brasileira era composta de $49 \%$ de homens, destes 46,8\% estavam em idade adulta (25-59 anos). Dados epidemiológicos demonstram que $60 \%$ das mortes registradas no Brasil eram de indivíduos do sexo masculino, sendo a principal causa de morte no período entre 2008 e 2010 os acidentes de trânsito (média anual de 112,3 mortes por 100.000 homens), seguida pelas neoplasias (média anual de 111,5 mortes por 100.000 homens) $)^{5}$.

As neoplasias de próstata representam o segundo câncer mais comum em homens, com estimativa de 1,1 milhōes de homens diagnosticados em 2012. A incidência apresenta variação de 25 vezes ao redor do mundo, sendo as maiores taxas encontradas na Austrália/Nova Zelândia (111,6 por 100.000), pois a prática de realizar o teste do PSA (Antígeno prostático específico, em português) tornou-se difundida na regiáo. As menores taxas dessa patologia sáo relatadas no Leste e Centro-Sul da Ásia $(10,5 \text { e } 4,5 \text { por } 100.000)^{6}$.

Com 307 mil mortes estimadas mundialmente em 2012, o câncer de próstata é a quinta causa de morte por câncer em homens $(6,6 \%$ do total de mortalidade masculina). Uma vez que o rastreio por PSA tem um efeito muito maior nas taxas de incidência quando comparado com seu efeito na taxa de mortalidade, as variações de taxa de mortalidade pelo mundo oscilam menos (entre 3 e 30 por 100.000). O número de mortes por câncer de próstata é maior em regióes menos desenvolvidas, quando comparado com as mais desenvolvidas $(165.000 \text { e } 142.000)^{6}$.

Somente na União Europeia, os custos com câncer atingiram 126 bilhóes de euros em 2009, onde as neoplasias de pulmáo representaram o mais alto custo econômico (18,8 bilhôes de euros - 15\% dos gastos com todas as neoplasias). O câncer de próstata representa o quarto na lista de custo $(8,43$ bilhóes de euros - 7\% $)^{7}$. Nos Estados Unidos da América, a estimativa total de gastos com o câncer de próstata em 2006 foi de 9,863 bilhóes de dólares, com uma média de custo anual por paciente de 10.612 dólares em fase inicial pós-diagnóstico, 2.134 dólares para cuidados contínuos e 33.691 dólares ao longo do último ano de vida. Apesar do declínio nas taxas de mortalidade, os custos tendem a subir devido ao aumento no número de diagnósticos, análises mais precoces e sobrevida aumentada ${ }^{8}$.

Em relação à prevalência nacional de câncer de próstata, de acordo com o relatório de 2014 do INCA (Instituto Nacional do Câncer), eram esperados 68.800 novos casos (70,42 por 100.000). Excluindo o câncer de pele não melanoma, o câncer de próstata é o mais incidente entre homens no país, sendo a região Sul a mais acometida $(91,24$ por 100.000$)$ seguida pelo Sudeste $(88,06 \text { por } 100.000)^{9}$.

De acordo com o Registro de Câncer de Base Populacional de São Paulo ${ }^{10}$, foram notificados 43.444 novos casos de câncer em 2011, com 19.537 casos ocorrendo somente em homens. No mesmo ano, o câncer de próstata representou 3.858 casos (26\% do total, excluindo o câncer de pele não melanoma). Distinto dos dados globais, o câncer de próstata é o mais comum em homens na cidade de Sáo Paulo, excluindo o câncer de pele não melanoma, representando $24,06 \%$ dos casos notificados entre 1997 e $2008^{10}$.

No Brasil, o aumento da expectativa de vida, o aprimoramento e desenvolvimento de métodos diagnósticos, a qualidade dos sistemas nacionais de informação, bem como a ocorrência de sobrediagnóstico 
devido à disseminação do rastreio por PSA e DRE (Digital Rectal Exam), podem explicar o aumento de incidência nos últimos anos ${ }^{9}$. Os fatores que determinam o aumento do risco de câncer de próstata náo foram integralmente elucidado ${ }^{11}$, porém, de acordo com a Sociedade Americana de Câncer (ACS) os fatores elencados atualmente são: Idade $(\mathrm{O}$ câncer de próstata é raro antes dos 40 anos, sendo que 6 em cada 10 casos ocorrem após os 65 anos); Etnia (A etnia afro-americana e indivíduos caribenhos com ancestralidade africana são as mais afetadas); Hereditariedade (Ter um pai ou irmão com câncer de próstata aumenta em mais de 2 vezes a chance de desenvolver esse tipo de neoplasia); Fator genético (Mutações dos genes BRCA1 e BRCA2 e portadores da síndrome de Lynch). Outros fatores também são propostos, como dieta, obesidade, tabaco, agentes químicos, inflamação da próstata, infecçóes sexualmente transmissíveis e vasectomia ${ }^{12}$. Com aumento da expectativa de vida mundialmente, espera-se que o número de novos casos de câncer de próstata atinja $60 \%$ em $2015^{9}$.

\section{Objetivos}

O estudo propóe-se a revisar e descrever o panorama atual das recomendaçóes relativas ao uso de rastreio populacional, seletivo ou oportunista de rotina para câncer de próstata mediante dosagem de níveis de PSA e/ou DRE. Adicionalmente, foram despendidos esforços no intuito de ressaltar a atual controvérsia de recomendaçóes existentes na literatura atual.

\section{Métodos}

O estudo consiste de revisão narrativa de literatura, usando os termos "Próstata e Neoplasia" e "PSA ou DRE" nas bases de dados MEDLINE (via PubMed), Embase, LILACS e Cochrane, conduzida na Universidade de Santo Amaro, São Paulo. As referências de cada estudo incluído foram checadas para potenciais citaçóes adicionais relevantes. O resultado desta estratégia de pesquisa encontra-se demonstrado na Tabela 1. A pesquisa foi atualizada em 21 de março de 2016.

Tabela 1: Resultados da estratégia de pesquisa adotada no estudo.

\begin{tabular}{|llcccc}
\hline$\#$ & Termos de pesquisa & PubMed & Embase & Cochrane & LILACS \\
1 & "Prostate" and "Neoplasia" and "Screening" & 66.230 & 4.680 & 28 & 156 \\
2 & "Screening" and "PSA" and "Digital Rectal Examination" & 2.610 & 6 & 19 & 29 \\
\hline 3 & $\# 1$ and \#2 & 1.027 & 1 & 11 & 7 \\
\hline \hline
\end{tabular}

Fonte: Elaborada pelos autores, 2016.

\section{Seleção de artigos}

A seleção inicial foi realizada com base na avaliação de títulos e resumos dos artigos identificados pela estratégia de pesquisa supracitada. Os resumos foram revisados por um único avaliador e, para aqueles que apresentaram compatibilidade com os critérios de elegibilidade, o artigo completo foi obtido para análise de inclusão no estudo.

Os critérios de inclusão foram:

- Artigos completos em língua inglesa.

- Artigos em língua nativa (português brasileiro) para aqueles oriundos de organizaçóes médicas brasileiras.

- Estudos controlados e randomizados (RCT) amplos e prospectivos ( $>10.000$ pacientes envolvidos).
- Meta-análises amplas, forças-tarefa nacionais, recomendaçôes de organizaçóes médicas nacionais.

Já os critérios de exclusão foram:

- Revisóes não sistemáticas, estudos observacionais, cartas, editoriais, comentários e relatos de caso ou séries de casos.

- Estudos em animal ou in vitro.

\section{Resultados}

A estratégia de pesquisa adotada gerou 1.046 citaçôes. Após cuidadosa avaliação dos títulos, resumos e recomendações de organizaçóes médicas/nacionais e consequente análise dos critérios de elegibilidade, foram selecionadas 25 referências para análise detalhada e apreciação crítica. 
Figura 1: Descrição do método de pesquisa

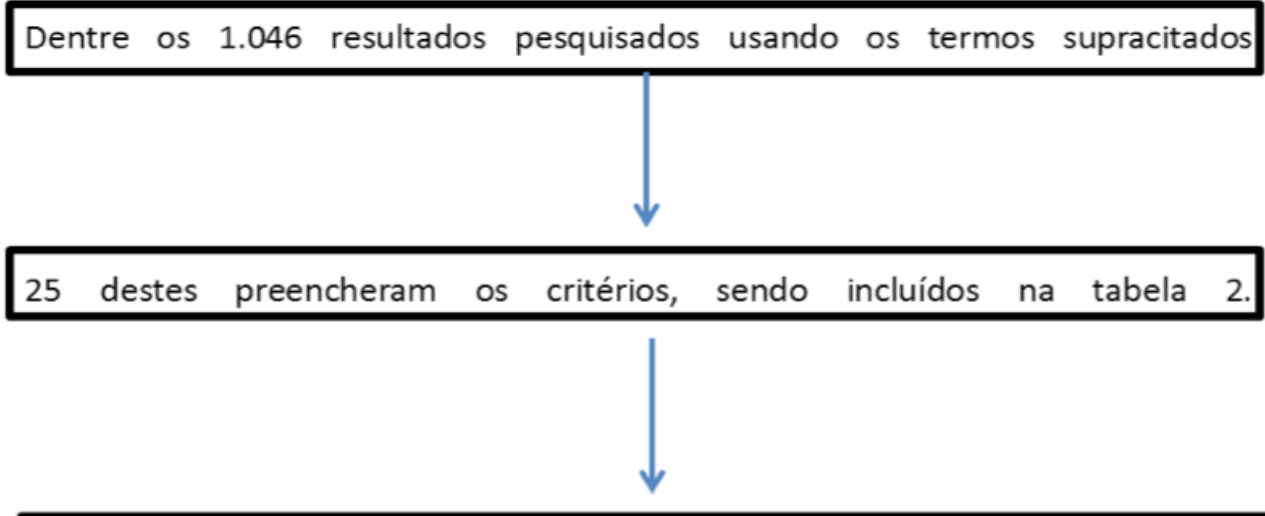

13 Instituições, RCT's ou meta-análises encontram-se representadas na tabela 2 .

Fonte: Elaborada pelos autores, 2016.

Os objetivos do rastreio contemplam a redução da mortalidade geral e específica associada à doença, e também o aprimoramento da qualidade de vida do indivíduo no futuro. Em relação ao câncer de próstata, a comunidade geral e a classe médica têm gerado notável debate sobre o rastreio, assim demonstrado por recomendações destoantes publicadas por organizaçóes médicas e políticas públicas de saúde ao redor do mundo. Dessa forma, propôs-se sumarizar as principais recomendaçôes encontradas na literatura com seus respectivos níveis de recomendação de cada instituição, na tentativa de atribuir-lhes o devido valor (Quadro 1 e Quadro 2).

Quadro 1: Recomendações mundiais em relação à metodologia de rastreio do câncer de próstata

\begin{tabular}{|c|c|c|c|}
\hline $\begin{array}{l}\text { Instituição ou } \\
\text { estudo }\end{array}$ & Recomendação & PSA - valor de corte & Intervalo de seguimento \\
\hline $\mathrm{SBU}^{13}$ & $\begin{array}{l}\text { Associar o PSA ao DRE no diagnóstico e manejo do } \\
\text { paciente com suspeita clínica de câncer de próstata. }\end{array}$ & $\geq 2,5 \mathrm{ng} / \mathrm{mL}$ ou $\geq 4,0 \mathrm{ng} / \mathrm{mL}$ & $\mathrm{n} / \mathrm{a}$ \\
\hline INCA $^{9}$ & $\begin{array}{l}\text { Não recomenda o rastreio para câncer de próstata. Foca } \\
\text { nos esforços para a prevenção primária. }\end{array}$ & $\mathrm{n} / \mathrm{a}$ & $\mathrm{n} / \mathrm{a}$ \\
\hline $\mathrm{EAU}^{14-17}$ & $\begin{array}{l}\text { Estratégia individualizada adaptada ao risco para a } \\
\text { detecção precoce deve ser oferecida a homens, bem } \\
\text { informados, com ao menos de } 10-15 \text { anos de expectativa } \\
\text { de vida. }\end{array}$ & $\begin{array}{l}\text { Teste precoce de PSA para } \\
\text { homens com risco elevado } \\
\text { (aqueles com PSA }>1 \mathrm{ng} / \\
\mathrm{mL} \text { aos } 40 \text { anos e }>2 \mathrm{ng} / \mathrm{mL} \\
\text { aos } 60 \text { anos) }\end{array}$ & $\begin{array}{l}\text { A cada } 2 \text { anos para aqueles em } \\
\text { risco ou a cada } 8 \text { anos para } \\
\text { aqueles fora de risco }\end{array}$ \\
\hline $\mathrm{MCS}^{18}$ & $\begin{array}{l}\text { Para homens com idade entre } 50-69 \text {. O teste de PSA } \\
\text { reduz a incidência de câncer de próstata metastático, } \\
\text { bem como as taxas de mortalidade específica. }\end{array}$ & $\mathrm{n} / \mathrm{a}$ & $\mathrm{n} / \mathrm{a}$ \\
\hline AUA $^{19}$ & $\begin{array}{l}\text { Recomendação contra o rastreio de PSA em homens } \\
\text { com menos de } 40 \text { anos. } \\
\text { Não recomenda o rastreio entre homens de risco médio } \\
\text { entre } 40-54 \text { anos. } \\
\text { Recomenda a tomada de decisão compartilhada para ho- } \\
\text { mens entre 55-69 anos que estejam considerando o ras- } \\
\text { treio com PSA, com base em seus valores e preferências. } \\
\text { Não recomenda o rastreio rotineiro de PSA em homens } \\
\text { com mais de } 70 \text { anos ou qualquer homem com menos } \\
\text { de } 10-15 \text { anos de expectativa de vida. }\end{array}$ & $\mathrm{n} / \mathrm{a}$ & $\begin{array}{l}\text { Rastreio com intervalo de } \geq 2 \\
\text { anos em aqueles que decidi- } \\
\text { ram rastrear após a tomada de } \\
\text { decisão compartilhada. }\end{array}$ \\
\hline
\end{tabular}


Quadro 1: Continuação.

\begin{tabular}{|c|c|c|c|}
\hline $\begin{array}{l}\text { Instituiçáo ou } \\
\text { estudo }\end{array}$ & Recomendação & PSA - valor de corte & Intervalo de seguimento \\
\hline $\mathrm{ACP}^{20}$ & $\begin{array}{l}\text { Rastrear homens entre 50-69 anos. O risco de câncer de } \\
\text { próstata, discussão de prós e contras, o estado geral do } \\
\text { paciente e expectativa de vida devem ser avaliadas. }\end{array}$ & $\begin{array}{l}\text { Níveis de PSA de } 2,5 \mathrm{ng} / \\
\mathrm{mL} \text { ou maiores requerem } \\
\text { avaliação anual }\end{array}$ & $\begin{array}{l}\text { Sem evidência de que rastrear a } \\
\text { cada }<4 \text { anos seja benéfico. }\end{array}$ \\
\hline $\mathrm{ACS}^{21,22}$ & $\begin{array}{l}\text { Homens assintomáticos ( }>50 \text { anos) com }>10 \text { anos de } \\
\text { expectativa de vida devem ter a oportunidade de tomar } \\
\text { uma decisão informada sobre o rastreio do câncer de } \\
\text { próstata. }\end{array}$ & $\begin{array}{l}\text { Considera o PSA > } 4,0 \mathrm{ng} / \\
\mathrm{mL} \text { e a tomada de decisão } \\
\text { individualizada quando o } \\
\text { PSA fica entre } 2,5 \text { e } 4,0 \mathrm{ng} / \\
\mathrm{mL}\end{array}$ & $\begin{array}{l}\text { PSA }<2,5 \mathrm{ng} / \mathrm{ml} \text {, rastreio a cada } \\
2 \text { anos. Se maior, rastreio anual }\end{array}$ \\
\hline CTFPHC $^{23,24}$ & Recomenda não rastrear com o PSA em qualquer idade. & $\mathrm{n} / \mathrm{a}$ & $\mathrm{n} / \mathrm{a}$ \\
\hline USPSTF $^{25,26}$ & $\begin{array}{l}\text { Recomendação contra os rastreios com base em PSA, } \\
\text { independentemente da idade. }\end{array}$ & $\mathrm{n} / \mathrm{a}$ & $\mathrm{n} / \mathrm{a}$ \\
\hline ERSPC $^{27-29}$ & $\begin{array}{l}\text { Confirma uma redução substancial }(21 \%) \text { na mortalida- } \\
\text { de por câncer de próstata, atribuível ao rastreio com PSA } \\
\text { após } 13 \text { anos de seguimento. }\end{array}$ & $3 \mathrm{ng} / \mathrm{mL}$ & $\mathrm{n} / \mathrm{a}$ \\
\hline $\mathrm{PLCO}^{30-33}$ & $\begin{array}{l}\text { Relata, após } 13 \text { anos de seguimento, não haver evidên- } \\
\text { cias de benefício na mortalidade para rastreio anual. }\end{array}$ & $4 \mathrm{ng} / \mathrm{mL}$ & $\mathrm{n} / \mathrm{a}$ \\
\hline $\operatorname{CDSR}^{34,35}$ & $\begin{array}{l}\text { Conclui que o rastreio náo reduz significantemente a } \\
\text { mortalidade específica por câncer de próstata. }\end{array}$ & $\mathrm{n} / \mathrm{a}$ & $\mathrm{n} / \mathrm{a}$ \\
\hline $\mathrm{NHS}^{36}$ & $\begin{array}{l}\text { Recomenda que o rastreio do câncer de próstata não seja } \\
\text { introduzido no Reino Unido no momento. Porém, ho- } \\
\text { mens que optarem pelo rastreio com PSA podem tomar } \\
\text { uma decisáo informada, baseada em informaçóes sobre } \\
\text { as vantagens e desvantagens de realizar o teste. }\end{array}$ & $\begin{array}{l}\text { Se o paciente decidir por } \\
\text { rastrear: } \\
50-59 \text { anos: } \geq 3,0 \mathrm{ng} / \mathrm{mL} 60 \text { - } \\
69 \text { anos: } \geq 4,0 \mathrm{ng} / \mathrm{mL} \geq 70 \\
\text { anos: }>5,0 \mathrm{ng} / \mathrm{mL}\end{array}$ & $\mathrm{n} / \mathrm{a}$ \\
\hline & 2016. & & \\
\hline
\end{tabular}

Quadro 2: Níveis de recomendação dos estudos selecionados.

\begin{tabular}{|c|c|c|}
\hline $\begin{array}{l}\text { Instituição } \\
\text { ou Estudo }\end{array}$ & Nível de recomendaçáo & Grau de recomendaçáo e evidência de robustez \\
\hline $\mathrm{SBU}^{13}$ & Grau de Recomendação: B & $\begin{array}{l}\text { A: Estudo experimental ou observacional de maior consis- } \\
\text { tência. } \\
\text { B: Estudo experimental ou observacional de menor consistên- } \\
\text { cia. } \\
\text { C: Relato de caso (estudo não controlado). } \\
\text { D: Opinião sem avaliação crítica, baseada em consenso, } \\
\text { estudo fisiológico ou modelo animal. }\end{array}$ \\
\hline INCA $^{9}$ & $\begin{array}{l}\text { Evidência científica de boa qualidade (Rastreio produz } \\
\text { mais danos que benefícios) }\end{array}$ & $\mathrm{n} / \mathrm{a}$ \\
\hline $\mathrm{EAU}^{14-17}$ & $\begin{array}{l}\text { Nível de evidência: } 3 \\
\text { Grau de recomendaçáo: } \mathrm{B}\end{array}$ & OCEBM Levels of Evidence Working Group ${ }^{37}$. \\
\hline $\mathrm{MCS}^{18}$ & Nível de evidência: 1 & OCEBM Levels of Evidence Working Group ${ }^{37}$. \\
\hline
\end{tabular}


Quadro 2: Continuação.

\begin{tabular}{|c|c|}
\hline $\begin{array}{l}\text { Instituiçáo } \\
\text { ou Estudo }\end{array}$ & Nível de recomendaçáo \\
\hline AUA $^{19}$ & $\begin{array}{l}\text { Contra o rastreio por PSA ( }<40 \text { anos }) \text { - Robustez de } \\
\text { evidência grau C. } \\
\text { Não recomenda rastreio entre } 40-54 \text { anos em homens } \\
\text { de risco médio - Robustez de evidência grau C. } \\
\text { Recomenda decisão compartilhada entre } 55-69 \text { anos } \\
\text { e proceder de acordo com os valores e preferências do } \\
\text { indivíduo - Robustez de evidência grau B. } \\
\text { Não recomenda rastreio por PSA em homens com }>70 \\
\text { anos ou com menos de 10-15 anos de expectativa de vida - } \\
\text { Robustez de evidência grau C. }\end{array}$ \\
\hline $\mathrm{ACP}^{20}$ & $\mathrm{n} / \mathrm{a}$ \\
\hline $\mathrm{ACS}^{21,22}$ & $\mathrm{n} / \mathrm{a}$ \\
\hline $\mathrm{CTFPHC}^{23,24}$ & $\begin{array}{l}\text { Homens com idade: } \\
\text { < } 55 \text { anos, não recomenda rastreio com PSA. } \\
\text { (Recomendação forte; Baixa qualidade de evidência). } \\
\text { 55-69 anos, não recomenda o rastreio com PSA } \\
\text { (Recomendação fraca; Moderada qualidade de evidência). } \\
\geq 70 \text { anos, não recomenda o rastreio com PSA. } \\
\text { (Recomendação forte; Baixa qualidade de evidência). }\end{array}$ \\
\hline
\end{tabular}

Grau de recomendaçáo e evidência de robustez

A estrutura de avaliação para qualidade de evidência é uma adaptaçáo e modificação da estrutura do GRADE (Grading of Recommendations, Assessment, Development and Evaluation). Nesta adaptação a AUA qualifica a evidência como alta, moderada ou baixa $(\mathrm{A}, \mathrm{B} \text { ou } \mathrm{C})^{38}$.

Seguiu o método AGREE II (Appraisal of Guidelines, Research and Evaluation in Europe) para produzir esta orientação - baseada nas diretrizes: $\mathrm{ACPM}^{20}, \mathrm{ACS}^{21,22}$ AUA $^{19}$ e USPSTF ${ }^{25,26}$.

Revisões sistemáticas foram avaliadas pelo comitê da ACS e deliberaçóes sobre a evidência ocorreram nos encontros do comitê e durante conferências.

Recomendações foram graduadas de acordo com o sistema GRADE $^{39}$

GRADE oferece dois níveis: forte e fraca. A qualidade de evidência é graduada como alta, moderada, baixa ou muito baixa. Baseia-se na probabilidade de estudos futuros alterarem a confiança do efeito estimado.

Grau A: Há grande certeza de que o benefício é substancial. Grau B: Há grande certeza de que o benefício é moderado, ou moderada certeza de que o benefício é moderado a substancial. Grau C: Os médicos podem fornecer esse serviço a pacientes selecionados, dependendo das circunstâncias individuais. Porém, para a maioria das pessoas sem sinais e sintomas, é provável que o serviço gere pequeno benefício.

Grau D: Há moderada ou alta certeza de que o serviço não gere benefício ou que os malefícios sejam maiores que os benefícios. Grau I - As evidências atuais são insuficientes para avaliar o benefício/malefício do serviço. As evidências são escassas, de baixa qualidade ou conflitantes e os benefícios/malefícios não podem ser determinados.

$\begin{array}{lll}\text { ERSPC }^{27-29} & \mathrm{n} / \mathrm{a} & \mathrm{n} / \mathrm{a} \\ \text { PLCO }^{30-33} & \mathrm{n} / \mathrm{a} & \mathrm{n} / \mathrm{a}\end{array}$

$\operatorname{CDSR}^{34,35} \mathrm{n} / \mathrm{a}$

A qualidade metodológica de três dos estudos foi avaliada como detentora de alto risco de viés. O ERSPC e o PLCO foram avaliados como detentores de baixo risco de viés, porém forneceram resultados contraditórios.

Até o momento não há dados no Reino Unido de estudos randomizados e controlados que demonstrem benefícios/ malefícios do uso do PSA como rastreio do câncer de próstata. Entretanto, há evidência da Europa que demonstram que o teste de PSA pode salvar vidas. Porém, é desconhecido quantos casos seriam diagnosticados e sobretratados.

Fonte: Elaborado pelos autores, 2016. 


\section{Discussão}

O rastreio do câncer de próstata representa um dos tópicos mais controversos em urologia. Por um lado, há dados de estudos randomizados revelando que o rastreio por PSA resulta em diagnósticos precoces, melhor desfecho clínico após tratamento e taxas de mortalidade reduzidas. No entanto, os pontos negativos incluem indicaçóes de biópsia desnecessárias devido a resultados falso-positivos nos exames de PSA, sobrediagnóstico de algumas neoplasias insignificantes e potencias efeitos colaterais da biopsia de próstata e/ ou do tratamento do câncer. A contínua controvérsia é destacada por recomendaçôes divergentes em relaçáo ao rastreio, oriundas de múltiplas organizações profissionais ${ }^{40}$.

Hayes e Barry ${ }^{41}$ revisaram as evidências atuais de estudos randomizados e relataram os que avaliaram o efeito do rastreio por PSA, comparando com a ausência de rastreio, no desfecho de mortalidade específica por câncer de próstata, e sugeriram uma abordagem que balanceasse os potenciais riscos e benefícios. De acordo com os autores, apenas homens que expressassem uma preferência definida por rastreio deveriam ser submetidos à dosagem de PSA ${ }^{41}$.

Existem várias possíveis explicaçóes para a ausência de redução de mortalidade em estudos como o PLCO (Prostate, Lung, Colorectal and Ovarian Cancer Screening Trial $^{30-33}$. Primeiro, o rastreio anual com teste de PSA, usando o valor de corte-padrão nos Estados Unidos de $4 \mathrm{ng} / \mathrm{mL}$ e DRE para indicar avaliação diagnóstica, pode não ser uma estratégia efetiva. No estudo ERSPC (European Randomised Study of Screening for Prostate Cancer) ${ }^{27-29}$, o valor de corte de PSA foi de $3 \mathrm{ng} / \mathrm{mL}$, com aumento potencial de sensibilidade, porém com redução de especificidade. No estudo $\mathrm{PLCO}^{30-33}$, um menor valor de corte poderia ter resultado no diagnóstico precoce de um maior número de cânceres de próstata. Adicionalmente, após 10 anos de seguimento, 312 pacientes do grupo de rastreio e 225 do grupo controle morreram por causas não relacionadas ao câncer de próstata e o excesso observado no grupo de rastreio foi possivelmente associado ao sobrediagnóstico do câncer de próstata ${ }^{32}$.

Um ponto de preocupação foi o substancial nível de "contaminação", ou uso de teste de PSA e DRE no grupo controle. Pinsky et al. ${ }^{42}$ forneceram uma descrição detalhada da contaminação no estudo $\mathrm{PLCO}^{30-33}$. Eventos de rastreio pré-estudo e educação universitária foram associados de maneira estatisticamente significante ao aumento nos níveis de contaminação. $\mathrm{O}$ valor médio estimado de contaminação por PSA (DRE) foi de 2,7 $(1,1)$ no grupo controle, comparado com 5,0 $(3,5)$ no grupo de rastreio.
Toda a controvérsia que existe neste tópico também é refletida na qualidade das evidências de recomendaçóes institucionais, descritas nos Quadros 2 e 3 . Algumas recomendaçóes individuais tal como o MCS (Melbourne Consensus Statement) são muito criticadas neste ponto. Ahmed $^{43}$ atentou que, apesar dos nobres objetivos, o MCS apresenta déficit de rigor, falhando em considerar a amplitude de evidência neste campo. Adicionalmente, às recomendaçóes de sociedades de especialistas, tais como a EAU (European Association of Urology) e a SBU (Sociedade Brasileira de Urologia), são atribuídos baixos níveis de evidência, uma vez que suas recomendaçóes são baseadas em estudos repletos de criticismo.

\section{Sobrediagnóstico e valor de corte do PSA}

A taxa de sobrediagnóstico do componente Finlandês, o maior no estudo ERSPC, foi relatada por Wu et al. ${ }^{44}$, ao observarem aproximadamente 3,4 casos por 100 homens rastreados.

Draisma et al. ${ }^{45}$ compararam dados de incidência dos Estados Unidos oriundos do programa SEER (Surveillance, Epidemiology and End Results) para estimar a fração de sobrediagnóstico de câncer de próstata devido ao rastreio com PSA entre homens (54-80 anos) no período de 1985 a 2000, combinados com os dados do estudo ERSPC, estimando taxas de sobrediagnóstico de $23-42 \%$, 42\% e 66\%, utilizando três modelos matemáticos distintos, para rastreio anual com valor de corte de $4 \mathrm{ng} / \mathrm{mL}$.

Embasada em ferramentas atualmente disponíveis, uma estratégia individualizada diagnosticará muitas lesōes insignificantes (acima de 50\% em estudos), das quais a maioria não necessitará qualquer forma de tratamento ativo. Isso se torna importante uma vez que a quebra do elo entre diagnóstico e tratamento ativo é o único meio de diminuir o sobretratamento, ao passo que se mantém o potencial benefício de diagnóstico precoce para homens que requererem o rastreio ${ }^{14}$.

Em relação ao $\mathrm{MCS}^{18}$, os autores descrevem a tomada de decisão compartilhada para o exame de PSA, no entanto, este método é de difícil aplicação na prática clínica regular. Outro ponto em discussão é que o diagnóstico do câncer de próstata deve ser dissociado da intervenção. Embora essa recomendação certamente seja lógica, a maioria dos médicos ainda indica intervençáo em muitos homens com doença de baixo risco promovendo danos e benefícios mínimos. A declaração 3 do MCS é similarmente vaga, estipulando que "o teste de PSA não deve ser considerado per se, mas sim como parte de uma abordagem multivariável na detecção precoce do câncer de próstata"18. Dezenas de nomogramas e calculadoras de risco existem, porém ainda 
não há evidência para sugerir sua aplicação na prática de rotina ${ }^{43}$.

Diante do exposto, observa-se que o rastreio seriado através da dosagem de PSA apresenta, no melhor dos cenários, um efeito modesto na mortalidade por câncer de próstata durante a primeira década de seguimento e este benefício vem acompanhado de custos, como o aumento substancial de sobrediagnóstico e sobretratamento. Adicionalmente, é importante relembrar que a questão principal não é saber quando o rastreio por PSA é efetivo, e sim quando ele produz mais benefício do que malefício ao paciente ${ }^{46}$.

\section{Conclusão}

Recomendaçóes discordantes em relação ao rastreio do câncer de próstata estão atualmente disponíveis, porém, a substancial inconsistência dos estudos randomizados e controlados realizados impossibilita a recomendação absoluta do rastreio com PSA para toda a população.

Existe grande cautela, principalmente no contexto de eventos adversos associados às consequências do sobrediagnóstico. Logo, estudos como os conduzidos por Vickers et al. ${ }^{16}$ e Carlsson et al. ${ }^{17}$ para determinar um valor de corte definitivo de PSA devem ser realizados, a fim de reduzir o sobrediagnóstico.

Conclui-se que a tomada de decisão compartilhada para homens assintomáticos de risco médio, com conscientização prévia do paciente, seja a via de escolha a ser adotada por políticas públicas de saúde, ao menos até que haja estudos de boa qualidade determinando a melhor metodologia e grupo populacional que se beneficiariam do rastreio do câncer de próstata. É digno de nota que o nível educacional da população pode influenciar na capacidade individual de tomada de decisão e este fator deve ser levado em consideraçáo.

Também deve-se esclarecer ao paciente que, mesmo que seja optado o rastreio por PSA, estudos recentes como o PIVOT ${ }^{47,48}$ e o PROTECT ${ }^{49,50}$ demonstraram que, após vinte anos de acompanhamento, manter o paciente em observação clínica ou tratar com cirurgia ou radioterapia não reduz a mortalidade, e ainda, entre as opçóes de tratamento, a prostatectomia tem o efeito mais negativo na função sexual e continência urinária. Conclui-se que os benefícios na deteç̧ão precoce estâo longe de ser uma realidade, em contraste com os malefícios que tendem a surgir precocemente e a persistir ao longo da sobrevida do paciente, especialmente após a cirurgia ou radioterapia.

Em suma, e alinhado com Pinsky et al. ${ }^{51}$, ainda não há definiçōes claras a respeito da utilidade e necessidade do rastreio por PSA, mesmo após mais de 25 anos de debate. Porém, os benefícios associados ao rastreio são possivelmente marginais, enquanto os malefícios certamente, substanciais. Dessa maneira, sob a ótica ética do princípio de não maleficência, o rastreio populacional em massa ainda não deve ser adotado como política pública de saúde.

\section{Referências}

1. Brasil. Ministério da Saúde. Secretaria de Atenção à Saúde. Departamento de Ações Programáticas Estratégicas. Política nacional de atenção integral à saúde da mulher: princípios e diretrizes. Brasília, DF: Ministério da Saúde; 2004.

2. Brasil. Ministério da Saúde. Secretaria de Atenção à Saúde. Departamento de Ações Programáticas Estratégicas. Política nacional de atenção integral à saúde do homem: princípios e diretrizes. Brasília, DF: Ministério da Saúde; 2009.

3. Carrara S, Russo JA, Faro L. A política de atenção à saúde do homem no Brasil: os paradoxos da medicalização do corpo masculino. Physis. 2009;19(3):659-78.

4. United Nations. Department of Economic and Social Affairs, Population Division. World Population Prospects: the 2015 revision [Internet]. New York: UN, 2015 [citado em 2016 jan 16]. Disponível em: <https://goo.gl/hTL2np>.

5. Knauth DR, Couto MT, Figueiredo WS. A visão dos profissionais sobre a presença e as demandas dos homens nos serviços de saúde: perspectivas para a análise da implantação da Política Nacional de Atenção Integral à Saúde do Homem. Ciênc Saúde Colet. 2012;17(10):2617-26.

6. Ferlay J, Soerjomataram I, Ervik M, Forman D, Bray F, Dikshit R, et al., editors. Globocan 2012: estimated cancer incidence, mortality and prevalence worldwide in 2012 [Internet]. Lyon: International Agency for Research on Cancer; 2013 [citado em 2015 abr 2]. Disponível em: <http://globocan.iarc.fr>.

7. Luengo-Fernandez R, Leal J, Gray A, Sullivan R. Economic burden of cancer across the European Union: a population-based cost analysis. Lancet Oncol. 2013;14(12):1165-74.

8. Roehrborn CG, Black LK. The economic burden of prostate cancer. BJU Int. 2011;108(6):806-13.

9. Instituto Nacional de Câncer José Alencar Gomes da Silva Coordenação de Prevenção e Vigilância. Estimativa 2014: incidência de câncer no Brasil [Internet]. Rio de Janeiro: Inca; 2014 [citado em 2015 nov 10]. Disponível em: $<$ https://goo.gl/p5GqAe>.

10. Departamento de Epidemiologia da Faculdade de Saúde Pública da USP. Registro de Câncer de Base Populacional (São Paulo) [Internet]. São Paulo, 2011 [citado em 2018 mar 27]. Disponível em: <https://goo.gl/pmd8kK>.

11. Richman EL, Kenfield SA, Stampfer MJ, Giovannucci EL, Chan JM. Egg, red meat, and poultry intake and risk of lethal 
prostate cancer in the prostate-specific antigen-era: incidence and survival. Cancer Prev Res (Phila). 2011;4(12):2110-21.

12. American Cancer Society. Prostate Cancer Risk Factors [Internet]. Atlanta; 2016 [citado em 2017 jul 27]. Disponível em: <https://goo.gl/1JrxEc>.

13. Nardi AC, Pompeo ACL, Faria EF, Guimaráes GC, Calixto JR, Ponte JRT, et al. Câncer de próstata: diagnóstico [Internet]. 2015 [citado em 2018 fev 2]. Disponível em: $<$ https://goo.gl/hLuAn2>.

14. Mottet N, Bellmunt J, Briers E, Bolla M, Bourke L, Cornford P, et al. Guidelines on Prostate Cancer [Internet]. Arnhem: European Association of Urology, 2015 [citado em 2015 nov 10]. Disponível em: <https://goo.gl/AprnEz>.

15. Kamangar F, Dores GM, Anderson WF. Patterns of cancer incidence, mortality, and prevalence across five continents: defining priorities to reduce cancer disparities in different geographic regions of the world. J Clin Oncol. 2006;24(14):2137-50.

16. Vickers AJ, Ulmert D, Sjoberg DD, Bennette CJ, Björk T, Gerdtsson A, et al. Strategy for detection of prostate cancer based on relation between prostate specific antigen at age 40-55 and long term risk of metastasis: case-control study. BMJ. 2013;346:f2023.

17. Carlsson S, Assel M, Sjoberg D, Ulmert D, Hugosson J, Lilja $\mathrm{H}$, et al. Influence of blood prostate specific antigen levels at age 60 on benefits and harms of prostate cancer screening: population based cohort study. BMJ. 2014;348:g2296.

18. Murphy DG, Ahlering T, Catalona WJ, Crowe H, Crowe J, Clarke N, et al. The Melbourne Consensus Statement on the early detection of prostate cancer. BJU Int. 2014;113(2):186-8.

19. Carter HB. American Urological Association (AUA) guideline on prostate cancer detection: process and rationale. BJU Int. 2013;112(5):543-7.

20. Qaseem A, Barry MJ, Denberg TD, Owens DK, Shekelle P. Screening for prostate cancer: a guidance statement from the Clinical Guidelines Committee of the American College of Physicians. Ann Intern Med. 2013;158(10):761-9.

21. Wolf AM, Wender RC, Etzioni RB, Thompson IM, D'Amico AV, Volk RJ, et al. American Cancer Society guideline for the early detection of prostate cancer: update 2010. CA Cancer J Clin. 2010;60(2):70-98.

22. American Cancer Society. American Cancer Society Guidelines for the Early Detection of Cancer. Prostate Cancer [Internet]. Atlanta; 2015 [citado em 2015 nov 10]. Disponível em: <https://goo.gl/soHGVB>.

23. Dunfield L, Usman A, Fitzpatrick-Lewis D, Shane A, editors. Screening for prostate cancer with prostate specific antigen (PSA) and treatment of early-stage or screen-detected prostate cancer: a systematic review of the clinical benefits and harms [Internet]. Ottawa: Canadian Task Force on Preventive Health Care; 2013 [citado em 2018 mar 27]. Disponível em: <https://goo.gl/amiiqi>.
24. Bell N, Connor Gorber S, Shane A, Joffres M, Singh H, Dickinson J. Recommendations on screening for prostate cancer with the prostate-specific antigen test. CMAJ. 2014;186(16):1225-34.

25. Chou R, Croswell JM, Dana T, Bougatsos C, Blazina I, Fu $\mathrm{R}$, et al. Screening for prostate cancer: a review of the evidence for the U.S. preventive services task force. Ann Intern Med. 2011;155(11):762-71.

26. Moyer VA; U.S. Preventive Services Task Force. Screening for prostate cancer: U.S. Preventive Services Task Force recommendation statement. Ann Intern Med. 2012;157(2):120-34.

27. Schröder FH, Hugosson J, Roobol MJ, Tammela TL, Ciatto S, Nelen V, et al. Screening and prostate-cancer mortality in a randomized European study. N Engl J Med. 2009;360(13):1320-8.

28. Schröder FH, Hugosson J, Roobol MJ, Tammela TL, Ciatto $S$, Nelen V, et al. Prostate-cancer mortality at 11 years of follow-up. N Engl J Med. 2012;366(11):981-90.

29. Schröder FH, Hugosson J, Roobol MJ, Tammela TL, Zappa M, Nelen V, et al. Screening and prostate cancer mortality: results of the European Randomised Study of Screening for Prostate Cancer (ERSPC) at 13 years of follow-up. Lancet. 2014;384(9959):2027-35.

30. Prorok PC, Andriole GL, Bresalier RS, Buys SS, Chia D, Crawford ED, et al. Design of the Prostate, Lung, Colorectal and Ovarian (PLCO) Cancer Screening Trial. Control Clin Trials. 2000;21(6 Suppl):273S-309S.

31. Andriole GL, Levin DL, Crawford ED, Gelmann EP, Pinsky PF, Chia D, et al. Prostate cancer screening in the Prostate, Lung, Colorectal and Ovarian (PLCO) Cancer Screening Trial: findings from the initial screening round of a randomized trial. J Natl Cancer Inst. 2005;97(6):433-8.

32. Andriole GL, Crawford ED, Grubb RL, Buys SS, Chia $\mathrm{D}$, Church TR, et al. Mortality results from a randomized Prostate-Cancer Screening Trial. N Engl J Med. 2009;360(13):1310-9.

33. Andriole GL, Crawford ED, Grubb RL, Buys SS, Chia D, Church TR, et al. Prostate cancer screening in the randomized Prostate, Lung, Colorectal, and Ovarian Cancer Screening Trial: mortality results after 13 years of follow-up. J Natl Cancer Inst. 2012;104(2):125-32.

34. Ilic D, O’Connor D, Green S, Wilt T. Screening for prostate cancer: a Cochrane systematic review. Cancer Causes Control. 2007;18(3):279-85

35. Ilic D, Neuberger MM, Djulbegovic M, Dahm P. Screening for prostate cancer. Cochrane Database Syst Rev. 2013;31(1):CD004720.

36. Burford DC, Kirby M, Austoker J. Prostate Cancer Risk Management Programme: information for primary care; PSA testing in asymptomatic men. Sheffield: NHS Cancer Screening Programmes; 2009. 
37. OCEBM Levels of Evidence Working Group. The Oxford 2011 Levels of Evidence [Internet]. Oxford: Oxford Centre for Evidence-Based Medicine; 2011 [citado em 2016 mar 20]. Disponível em: <https://goo.gl/ujFajw>.

38. Atkins D, Best D, Briss PA, Eccles M, Falck-Ytter, Flottorp $\mathrm{S}$, et al. Grading quality of evidence and strength of recommendations. BMJ. 2004;328(7454):1490.

39. Schünemann H, Brozek J, Guyatt G, Oxman A, editors. GRADE handbook for grading quality of evidence and strength of recommendations. [S.1.]: The GRADE Working Group; 2009.

40. Loeb S. Guideline of guidelines: prostate cancer screening. BJU Int 2014;114(3):323-5.

41. Hayes JH, Barry MJ. Screening for prostate cancer with the prostate-specific antigen test: a review of current evidence. JAMA. 2014;311(11):1143-9.

42. Pinsky PF, Blacka A, Kramer BS, Miller A, Prorok PC, Berg C. Assessing contamination and compliance in the prostate component of the Prostate, Lung, Colorectal, and Ovarian (PLCO) Cancer Screening Trial. Clin Trials. 2010;7(4):303-11.

43. Ahmed, HU. Prostate cancer: Melbourne consensus-noble but misguided. Nat Rev Urol. 2014;11(5):250-1.

44. Wu GH, Auvinen A, Määttänen L, Tammela TL, Stenman UH, Hakama M, et al. Number of screens for overdetection as an indicator of absolute risk of overdiagnosis in prostate cancer screening. Int J Cancer. 2012;131(6):1367-75.
45. Draisma G, Etzioni R, Tsodikov A, Mariotto A, Wever E, Gulati R, et al. Lead time and overdiagnosis in prostate-specific antigen screening: importance of methods and context. J Natl Cancer Inst. 2009;101(6):374-83.

46. Barry MJ. Screening for prostate cancer - the controversy that refuses to die. N Engl J Med. 2009;360:1351-4.

47. Wilt TJ, Jones KM, Barry MJ, Andriole GL, Culkin $\mathrm{D}$, Wheeler $\mathrm{T}$, et al. Follow-up of prostatectomy versus observation for early prostate cancer. N Engl J Med. 2017;377(2):132-42.

48. Wilt TJ, Brawer MK, Barry MJ, Jones KM, Kwon Y, Gingrich JR, et al. The prostate cancer intervention versus observation trial: VA/NCI/AHRQ cooperative studies program \#407 (PIVOT): design and baseline results of a randomized controlled trial comparing radical prostatectomy to watchful waiting for men with clinically localized prostate cancer. Contemp Clin Trials. 2009;30(1):81-7.

49. Donovan JL, Hamdy FC, Lane JA, Mason M, Metcalfe C, Walsh E, et al. Patient-reported outcomes after monitoring, surgery, or radiotherapy for prostate cancer. N Engl J Med. 2016;375(15):1425-37.

50. Donovan J, Hamdy F, Neal D, Peters T, Oliver S, Brindle $\mathrm{L}$, et al. Prostate Testing for Cancer and Treatment (ProtecT) feasibility study. Health Technol Assess 2003;7(14):1-88.

51. Pinsky PF, Prorok PC, Kramer BS. Prostate cancer screening - a perspective on the current state of the evidence. $\mathrm{N}$ Engl J Med. 2017;376(13):1285-89.

\section{Como citar este artigo:}

Betiol JC, Nascimento CD, Silva FAR, Carabetta Júnior V, Hachul M. Rastreio do câncer de próstata: revisão sistemática da literatura sobre as perspectivas mundiais. Rev. Aten. Saúde. 2018;16(55):89-98. 